Journal of the Operations Research

Society of Japan

Vol. 43, No. 3, September 2000

\title{
AN INTEGRATED ANALYTICAL/SIMULATION APPROACH FOR ECONOMIC DESIGN OF AN AGV SYSTEM
}

\author{
Masaru Nakano \\ Toyota Central R\&D Labs., Inc.
}

\author{
Katsuhisa Ohno \\ Nagoya Institute of Technology
}

(Received April 14, 1999; Final February 14, 2000)

\begin{abstract}
This paper proposes an integrated analytical/simulation approach for designing an automated guided vehicle system (AGVS) which consists of AGVs, machines with input buffers and a dispatching station in a just-in-time (JIT) environment. The objective is to determine the number of AGVs, the input buffer capacities and locations of the machines that minimize a cost function under the constraint that the planned utilization of each machine is achieved.

The integrated analytical/simulation approach employs a simulation model to evaluate the performance of the AGVS and an analytical approach to reduce the repetition number of simulations in searching an optimal solution. The analytical approach leads to an efficient iterative procedure based on monotonicity properties of the cost function and the machine utilization in each design factor, and lower bounds of the number of AGVs and the input buffer capacities. Moreover, initial locations of the machines are derived from the HLP inequality. Computational results are given to demonstrate the efficiency of the proposed procedure. It is observed that the lower bounds and the initial locations are the optimal solution in case of deterministic processing times at machines.
\end{abstract}

\section{Introduction}

Traditional approaches to modeling manufacturing systems can be categorized as either analytical based or simulation based (Mahadevan and Narendran 1993). Analytical approaches which model the systems by means of mathematical equations often need unrealistic assumptions, while simulation models are often time-consuming and do not provide exact solutions. Shantikumar and Sargent (1983) defined four hybrid analytical/simulation models. Their analytical and simulation models are developed independently, and their corresponding solution procedures are combined in problem solving. Class four type in their hybrid models is defined as a model in which a simulation model is used as an overall model of the total system, and it requires values from the solution procedure of the analytic model of a portion of the system for some or all of its parameters. Most of conventional hybrid approaches employ a simulation model as an overall model of the total system and an analytical model only for determining some of its initial parameters of the simulation model. However, the conventional approaches are not sufficient to reduce the repetition number of simulations after setting the initial parameters.

In many problems of designing manufacturing systems, the cost functions and constraints have monotone structure in design parameters. For example, as the number of facilities increases, both the total cost and production rate of the system increase. As the buffer size between machines increases, the production rate of the system increases. As the performance of facilities becomes higher, the makespan becomes shorter. Such monotone structure has been considered in analytical approaches (Buzacott and Shanthikumar 1993, Glasserman and Yao 1994). This paper proposes an integrated analytical/simulation approach which 
is based on the monotonicity properties. The monotone structure makes a tree search algorithm efficient and gives initial design factors for the optimization. The integrated idea using analytical properties of manufacturing systems described in this paper is not included in the hybrid approaches defined by Shantikumar and Sargent(1993). Thus, we use "integrated" instead of "hybrid".

The problem studied in this paper is to design an automated guided vehicle system (AGVS). AGVSs are popular in modernized factories to utilize workstations or machines effectively under changes in demands. The design of AGVSs is a complex task since a design engineer has to take into account the following design factors which many researches have addressed (for example, Sinriech and Tanchoco 1992, Egbelu 1993, Kim and Tanchoco 1993, Hwang et al. 1996, Egbelu and Tanchoco 1984, Occeña and Yokota 1991, Kim and Klein 1996) :

(1) Number of AGVs

(2) Guided path layout

(3) Unit load size

(4) Dispatching rule

(5) Buffer capacity

(6) Location of workstations or placement of pickup and delivery points

Mahadevan and Narendran (1993) and Malmborg (1994) considered the trade-offs between those factors using hybrid analytical/simulation models. Their overall model is a simulation model and an analytical model is used to estimate a starting point of design factors in the simulation solution procedure. Maxwell and Muckstadt (1982), Egbelu (1987), and Nahadevan and Narendran (1993) estimated the initial number of AGVs by evaluating empty and loaded trips of AGVs.

The AGVS studied in this paper consists of AGVs, machines with input buffers and a dispatching station in a just-in-time (JIT) environment. The objective is to determine the number of AGVs, the input buffer capacities and locations of the machines that minimize a cost function under the constraint that the planned utilization of each machine is achieved. An example of AGV loop configuration is illustrated in Figure 1. Such AGVSs are popular in mass production systems. Since travel times of AGVs are constant, the AGVS can not be analyzed rigorously by the queueing theory. Johnson and Brandeau (1993) considered an AGVS in the JIT environment which had no buffer capacities in front of machines, and cost function and constraints different from those in this paper, and formulated it as a binary integer programming problem and an $\mathrm{M} / \mathrm{G} / \mathrm{c}$ queueing system. That is, they used only an analytical approach. Malmborg (1994) considered a looped AGVS which had a vehicle initiated dispatching rule instead of the JIT dispatching rule, and employed a hybrid analytical/simulation approach with two random search procedures: a greedy and a simulated annealing procedures.

This paper presents an integrated analytical/simulation approach, which leads to an efficient iterative procedure based on the monotonicity properties that the cost function and the utilization of each machine are increasing in the number of AGVs and the buffer capacities. The procedure uses tight lower bounds of the number of AGVs and the buffer capacities which are obtained analytically from the planned machine utilizations. The theory of majorization(Marshall and Alkin 1979) is used to set the initial locations of machines. Computational results are given to demonstrate the efficiency of the proposed procedure.

The organization of the paper is as follows. Section 2 describes the AGVS and a cost function of the number of AGVs, the buffer capacities and the locations of workstations as 


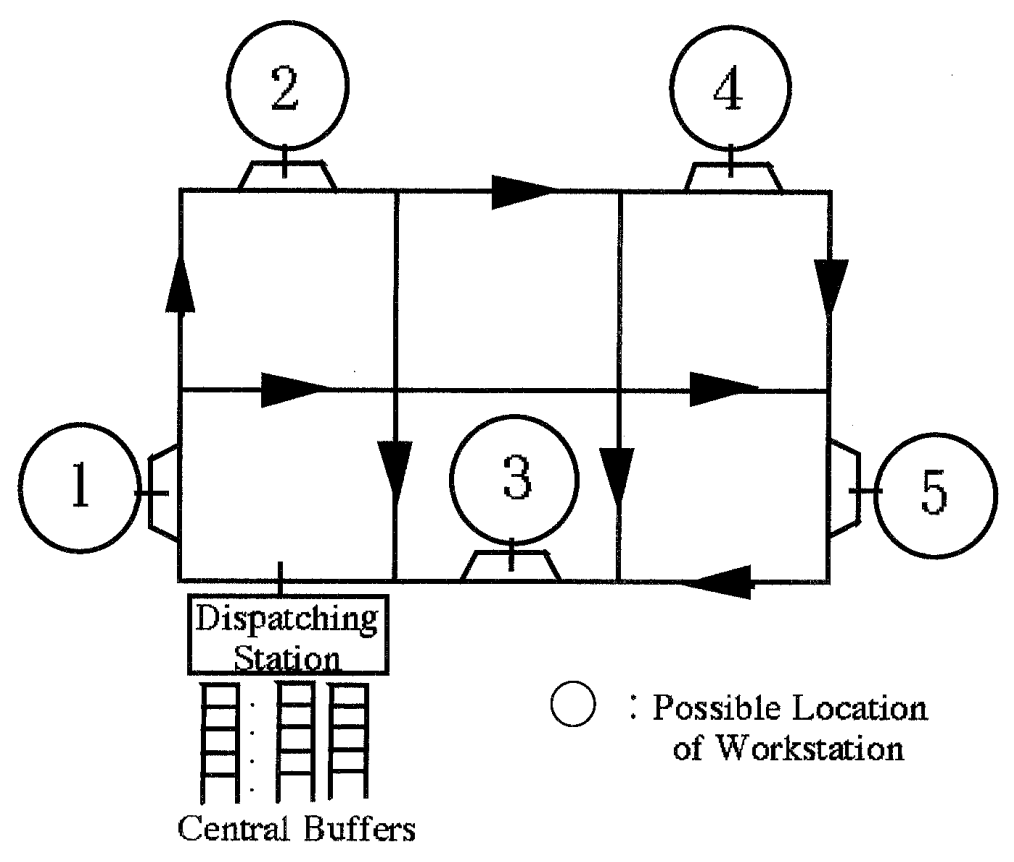

Figure 1: Example of AGV loop configuration

design factors. In section 3 the lower bounds of the number of AGVs and the buffer capacities are derived analytically. The optimal locations of the workstations for the lower bounds are also presented. Moreover, we prove the monotonicity properties of the cost function and the machine utilization in each of the design factors. Section 4 proposes an iterative solution procedure integrating the monotonicity properties and simulation. Numerical results are given in section 5 .

\section{Statement of Problem}

We consider the AGVS in the JIT environment, which consists of AGVs, a dispatching station, and several workstations. The workstations are numbered 1 through $N$ and process $N$ types of parts. Workstation $i$ consists of machine $i$ and input buffer $i(i=1,2, \cdots, N)$. Part $i$ is processed only at machine $i$. Parts processed are assumed to be instantaneously extruded outside the system without blocking.

AGVs deliver part $i$ to workstation $i$ and are assumed to carry one unit at a time. The number of AGVs is denoted by $M$. All AGVs are unidirectional and have the same performance. The possible locations where the workstations can be placed are numbered 1 through $L(\geq N)$. The delivery time when any AGV goes from the dispatching station to location $j$ is denoted by $\nu_{j}$. The delivery time includes the pickup time at the dispatching station and the deposit time at the workstation. The return time of any AGV from location $j$ to the dispatching station is denoted by $\xi_{j}$. Then the total travel time denoted by $\eta_{j}$ is given by $\nu_{j}+\xi_{j}$. All $\nu_{j}, \xi_{j}$ and $\eta_{j}$ are assumed to be constant. The waiting times to avoid collisions at the intersections of the routes are assumed to be insignificant. In addition, it is assumed that AGVs are not blocked by other AGVs because AGV routes have the unloading routes as shown in Figure 1 . Therefore, $\eta_{j}, \nu_{j}$ are constant.

It is assumed that the dispatching station has $\mathrm{N}$ central buffers. Part $i$ is stored in central buffer $i$. The distribution of the processing times of part $i$ at machine $i$ is generally distributed with mean $1 / \mu_{i}$. The capacity of the input buffer in front of machine $i$ is denoted 
by $b_{i}$. In the JIT environment, when the level of component inventory at an input buffer falls below a threshold level, a delivery order is placed at the dispatching station. JIT systems are known to have the ability to adapt to changes in demands while maintaining greatly reduced work-in-process inventories (Monden 1993, Ohno et al. 1995). Machine $i$ is referred to as a withdrawing machine if its order is not yet filled. Since the unit load size of an AGV is one, the threshold level can be set as $b_{i}$ without loss of generality. The threshold level of the input buffer at the machine equals to the number of withdrawal kanbans of the machine.

Let $u_{i}$ be the utilization of machine $i$ and $\widehat{u}_{i}$ the planned utilization of machine $i$ that is determined from a forecast demand of the completed components of machine $i$. The utilization $u_{i}$ is a nonlinear function of decision factors described below. The value of $u_{i}$ is obtained by simulation in this paper, because the queueing theory can not solve the AGVS with constant travel times of AGVs. The components are dispatched to the withdrawing machine that has the maximum value of $\widehat{u}_{i}-u_{i}$ among withdrawing machines.

We consider the problem of determining the number of $A G V s$, the buffer capacities and locations of the workstations. In this problem, the throughput of completed components at each workstation is required to achieve a forecast demand. Therefore, the objective is to minimize a cost function subject to the forecast demand constraint. The following notation is used to describe the cost function:

$C_{A}=$ fixed unit cost of an $\mathrm{AGV}$, which includes the investment cost

$C_{B}=$ fixed cost per unit capacity of input buffer, which includes the space, initial setting and maintenance costs

$C_{D}=$ fixed travel cost per distance unit on the guide path. It corresponds to the operating cost of AGVs and includes the batteries, recharge and maintenance costs.

$D_{i}=$ forecast demand per unit time for completed components of part $i$

$x_{i j}=1$ if workstation $i(i=1, \cdots, N)$ is located at location $j(j=1, \cdots, L)$ and 0 otherwise

$\tilde{x}=$ location matrix $\left(x_{i j}\right), i=1, \cdots, N, j=1, \cdots, L$

$\bar{b}=$ buffer capacity vector $\left(b_{1}, \cdots, b_{N}\right)$

The cost function is denoted by $\Psi(M, \vec{b}, \tilde{x})$. The objective is to minimize the sum of costs of AGVs, input buffers and locations of workstations under the constraint that the planned utilization of each machine is achieved, namely,

$$
\begin{aligned}
& \min _{M, \tilde{b}, \tilde{x}} \Psi(M, \bar{b}, \tilde{x})=C_{A} M+C_{B} \sum_{i=1}^{N} b_{i}+C_{D} \sum_{i=1}^{N}\left(D_{i} \sum_{j=1}^{L} \eta_{j} x_{i j}\right) \\
& \text { subject to } \\
& \quad u_{i}(M, \bar{b}, \tilde{x}) \geq \widehat{u}_{i}, \quad i=1, \cdots, N \\
& M: \text { positive integer, } \\
& \quad b_{i}: \text { non-negative integer, } \quad i=1, \cdots, N \\
& x_{i j}=0 \text { or } 1, \quad i=1, \cdots, N, \quad j=1, \cdots, L \\
& \sum_{j=1}^{L} x_{i j}=1, \quad i=1, \cdots, N, \\
& \sum_{i=1}^{N} x_{i j} \leq 1, \quad j=1, \cdots, L .
\end{aligned}
$$


Note that the throughput of completed components of part $i$ equals to $u_{i}(M, \bar{b}, \tilde{x}) \mu_{i}(i=$ $1, \cdots, N)$. Thus, constraint (2) is obtained from $u_{i}(M, \bar{b}, \tilde{x}) \mu_{i} \geq D_{i}=\widehat{u}_{i} \mu_{i}(i=1, \cdots, N)$. The problem seems essential in designing mass production systems in such as automobile industry.

Let $\Omega$ be the set of $(M, \bar{b}, \tilde{x})$ which satisfies constraint $(2),\left(M^{*}, \bar{b}^{*}, \tilde{x}^{*}\right) \epsilon \Omega$ be the vector of $(M, \bar{b}, \tilde{x})$ that minimizes $\Psi(M, \bar{b}, \tilde{x})$, and $\Psi^{*}$ be the minimum value.

\section{Properties of Problem}

\subsection{Lower bounds}

At first, we consider the lower bound of $M$. Let $E_{\text {home }}$ be the average number of AGVs waiting at the dispatching station and $T H_{i}$ be the throughput at workstation $i$. Once workstation $i$ is placed at some location, the delivery time and the total travel time are fixed and denoted by $g_{i}$ and $d_{i}$, respectively. Since the total travel time for an AGV to go to workstation $i$ is $d_{i}$ and $T H_{i}$ components per unit time must be delivered, the average number of AGVs going to or returning from workstation $i$ is equal to $T H_{i} d_{i}$ from Little's formula (see, for example, page 50 in Buzacott and Shanthikumar 1993). Consequently, the following equation holds:

$$
E_{\text {home }}+\sum_{i=1}^{N} T H_{i} d_{i}=M
$$

It follows from $E_{\text {home }} \geq 0, T H_{i}=u_{i}(M, \bar{b}, \tilde{x}) \mu_{i}$ and constraint (2) that

$$
M \geq \sum_{i=1}^{N} \widehat{u}_{i} \mu_{i} d_{i} \text {. }
$$

Let $M_{L B}$ be the lower bound of $M$ under constraint (2). Then it follows that

$$
M_{L B}=\left\lceil\sum_{i=1}^{N} \widehat{u}_{i} \mu_{i} d_{i}\right\rceil
$$

where $\lceil x\rceil$ is the smallest integer greater than or equal to $x$.

Next, we deal with the lower bound of $b_{i}, i=1, \cdots, N$. Suppose an extreme case where machine $i$ has just completed a processing when AGVs enough to serve the calls from workstation $i$ wait at the dispatching station. Then machine $i$ gets a component from the input buffer and calls an AGV for delivery. Since the components consumed by machine $i$ during the AGV delivery time to machine $i$ are given by $\widehat{u}_{i} \mu_{i} g_{i}, b_{i}$ must satisfy the following inequality:

$$
b_{i} \geq \widehat{u}_{i} \mu_{i} g_{i}, \quad i=1, \cdots, N .
$$

Then the lower bound, $b_{i L B}$, of $b_{i}$ under constraint (2) is given by

$$
b_{i L B}=\left\lceil\widehat{u}_{i} \mu_{i} g_{i}\right\rceil, \quad i=1, \cdots, N .
$$

\subsection{Optimal location}

We consider optimal locations of workstations when $M$ and $\bar{b}$ are given by the lower bounds $M_{L B}$ and $\bar{b}_{L B}$, respectively, where $\bar{b}_{L B}=\left(b_{1 L B}, \cdots, b_{N L B}\right)$. Equations (4) and (6) are rewritten using $d_{i}=\sum_{j=1}^{L} \eta_{j} x_{i j}$ and $g_{i}=\sum_{j=1}^{L} \nu_{j} x_{i j}, \quad i=1, \cdots, N$ as follows:

$$
\begin{aligned}
& M_{L B}=\left\lceil\sum_{i=1}^{N} \sum_{j=1}^{L} \widehat{u}_{i} \mu_{i} \eta_{j} x_{i j}\right\rceil, \\
& b_{i L B}=\left\lceil\sum_{j=1}^{L} \widehat{u}_{i} \mu_{i} \nu_{j} x_{i j}\right\rceil, \quad i=1, \cdots, N .
\end{aligned}
$$


From cost function (1) and $D_{i}=\widehat{u}_{i} \mu_{i}(i=1, \cdots, N)$, we consider the following lower bound function $\Psi_{L B}$ :

$$
\Psi\left(M_{L B}, \bar{b}_{L B}, \tilde{x}\right) \geq \sum_{i=1}^{N} \sum_{j=1}^{L} \widehat{u}_{i} \mu_{i}\left\{\left(C_{A}+C_{D}\right) \eta_{j}+C_{B} \nu_{j}\right\} x_{i j} \stackrel{\text { def }}{=} \Psi_{L B}(\tilde{x}) .
$$

The well known HLP inequality derived from the theory of majorization( for example, Proposition A.3 in Marshall and Alkin 1979) is that if $a_{i}$ and $c_{i}, i=1, \cdots, N$, are two sets of numbers, then

$$
\sum_{i=1}^{N} a_{(i)} c_{[i]} \leq \sum_{i=1}^{N} a_{i} c_{i} \leq \sum_{i=1}^{N} a_{(i)} c_{(i)}
$$

where $a_{(i)}$ and $c_{(i)}, i=1, \cdots, N$ denote the elements rearranged in decreasing order and $c_{[i]}, i=1, \cdots, N$ denote the elements rearranged in increasing order. Similarly, the elements of $\left\{\widehat{u}_{i} \mu_{i}, i=1, \cdots, N\right\}$ in decreasing order are denoted by $(\widehat{u} \mu)_{(i)}$ and the $j$ th elements of $\left\{\left(C_{A}+C_{D}\right) \eta_{j}+C_{B} \nu_{j}, j=1, \cdots, N\right\}$ in increasing order are denoted by $\left(\left(C_{A}+C_{D}\right) \eta+C_{B} \nu\right)_{[j]}$. Then the HLP inequality implies

$$
\Psi_{L B}(\tilde{x}) \geq \sum_{i=1}^{N}(\widehat{u} \mu)_{(i)}\left(\left(C_{A}+C_{D}\right) \eta+C_{B} \nu\right)_{[i]} .
$$

Therefore, the location matrix $\tilde{x}_{L B}$ that minimizes $\Psi_{L B}(\tilde{x})$ is obtained by the following rule:

$$
\begin{aligned}
& x_{(i)[j]}= \begin{cases}1 & \text { if }(i)=[j], \\
0 & \text { if }(i) \neq[j],\end{cases} \\
& i=1, \cdots, N, \quad j=1, \cdots, L .
\end{aligned}
$$

Equation (12) means that workstation (i) in increasing order of $\left\{\widehat{u}_{j} \mu_{j}, j=1, \cdots, N\right\}$ should be located at location [i] in decreasing order of $\left\{\left(C_{A}+C_{D}\right) \eta_{j}+C_{B} \nu_{j}, j=1, \cdots, N\right\}$ Note that locations [1], [2], $\cdots,[N]$ are used and the other locations $[N+1],[N+2], \cdots,[L]$ are discarded.

\subsection{Monotonicity}

We prove the monotonicity properties of the cost function and the machine utilization. It is clear from cost function (1) that $\Psi(M, \bar{b}, \tilde{x})$ is increasing in $M, b_{i}, i=1, \cdots, N$ and $\eta_{j}, j=1, \cdots, L$.

The monotonicity properties of the machine utilization are given in the following theorem:

Theorem 1 The machine utilization $u_{i}(M, \vec{b}, \tilde{x}), i=1, \cdots, N$, is increasing in $M$ and $b_{i}, i=$ $1, \cdots, N$, respectively, and decreasing in $g_{i}$.

Proof. The following notation is used only in this proof:

For $i=1, \cdots, N$ and $n=1,2, \cdots$,

$A_{i}(n)=$ the arrival epoch of the $n$th component at the buffer of workstation $i$,

$D_{i}(n)=$ the delivery order epoch to the dispatching station of the nth component of machine $i$,

$E(X)=$ expectation of random variable $X$,

$S_{i}(n)=$ the processing time including loading/unloading time of the $n$th component at machine $i$,

$T_{i}(n)=$ the completion epoch of processing the $n$th component at machine $i$,

$W_{i}(n)=$ the waiting time at the dispatching station of the nth component at machine $i$ for an AGV which delivers it to machine $i$. 
Since the machine utilization can be rewritten as

$$
u_{i}(M, \bar{b}, \tilde{x})=\lim _{n \rightarrow \infty} n /\left\{\mu_{i} E\left(T_{i}(n)\right)\right\},
$$

it sufficies to prove that $T_{i}(n)$ is decreasing in $M$ and $b_{i}$, respectively, and increasing in $g_{i}$. Suppose that all buffers are full at time 0 . Then

$$
A_{i}(n)=D_{i}(n)=W_{i}(n)=0 \quad \text { for } n=1, \cdots, b_{i}, i=1, \cdots, N .
$$

Since the processing of the nth component at machine $i$ can start after it arrives at machine $i$ and the processing of the $(n-1)$ th component completes, it holds that

$$
T_{i}(n)=S_{i}(n)+\max \left\{T_{i}(n-1), A_{i}(n)\right\},
$$

where $T_{i}(0)=0$. The delivery order of the $\left(n+b_{i}\right)$ th component is placed at $T_{i}(n)$. That is, for $n=b_{i}+1, b_{i}+2, \cdots$,

$$
D_{i}(n)=T_{i}\left(n-b_{i}\right)
$$

Since the $n$th component at machine $i$ is dispatched at $D_{i}(n)+W_{i}(n), A_{i}(n)$ is expressed as

$$
A_{i}(n)=D_{i}(n)+W_{i}(n)+g_{i} .
$$

Equations (15) and (17) lead to

$$
T_{i}(n)=S_{i}(n)+\max \left\{T_{i}(n-1), D_{i}(n)+W_{i}(n)+g_{i}\right\}
$$

The dispatching rule of $A G V s$ is to dispatch a component to the machine that maximizes $\left(\widehat{u}_{i}-u_{i}\right)$ among the withdrawing machines. This rule can be implemented in the following way: Delivery orders from each machine wait for AGVs in the arrival order in each separate queue at the dispatching station. When the delivery order of the nth component at machine $i$ arrives at the dispatching station to find the delivery orders of the $(n-l)$ th, $\cdots,(n-1)$ th components waiting in the queue, the value of $\left(\widehat{u}_{i}-u_{i}\right)$ is given by $\max _{i}\left\{\widehat{u}_{i}-\left(\sum_{k=1}^{m-b_{i}} S_{i}(k)\right) / D_{i}(m) ; m=n, \cdots, n-l\right\}$. When an $A G V$ returns to the delivery station, it picks up a component for the queue with the maximum value of $\left(\widehat{u}_{i}-u_{i}\right)$ among withdrawing machines and delivers it to the machine.

This rule determines the dispatching order $D(k) ; k=1,2, \cdots$ derived from all delivery orders $\left\{D_{i}(n) ; n=b_{i}+1, \cdots, i=1, \cdots, N\right\}$. For example, $D(1)=\min _{i=1, \cdots, N}\left\{D_{i}\left(b_{i}+1\right)\right\}$. Define the transformations $(i(k), n(k))$ and $k(i, n)$ by

$$
D(k)=D_{i(k)}(n(k)) \quad \text { and } \quad D_{i}(n)=D(k(i, n)),
$$

that is, $(i(k), n(k))$ means that the $k$ th delivery order epoch is the $n(k)$ th delivery order epoch of machine $i(k)$, and conversely $k(i, n)$ means that the nth delivery order of machine $i$ is the $k(i, n)$ th delivery epoch. Then the waiting time $W_{i}(n)$ can be expressed as

$$
\begin{gathered}
W_{i}(n)=W(k(i, n))=\max \left\{0, \underset{l=1, \cdots, k(i, n)-1}{M}\left\{D(l)+W(l)+d_{i(l)}\right\}-D(k(i, n))\right\}, \\
n=b_{i}+1, b_{i}+2, \cdots,
\end{gathered}
$$

where $\max _{1, \cdots, m}^{M}\left\{y_{l}\right\}$ denotes the $M$ th largest value of the set of real numbers $\left\{y_{l}\right\}$.

Therefore, by equations (16) and (18),

$$
\begin{gathered}
T_{i}(n)=S_{i}(n)+\max \left\{T_{i}(n-1), g_{i}+\max \left\{T_{i}\left(n-b_{i}\right),\right.\right. \\
\left.\left.\max _{l=1, \cdots, k(i, n)-1}^{M}\left\{D(l)+W(l)+d_{i(l)}\right\}\right\}\right\} .
\end{gathered}
$$

Since $\max _{l=1, \cdots, k(i, n)-1}^{M}\left\{D(l)+W(l)+d_{i(l)}\right\}$ and $T_{i}(n-1)$ are decreasing in $M$ and $b_{i}$, respectively, equation (21) implies that $T_{i}(n)$ is decreasing in $M$ and $b_{i}$, respectively, and increasing in $g_{i}$. The proof is concluded.

Theorem 1 implies that for any $(M, \bar{b}, \tilde{x}) \epsilon \Omega$, it holds that $(M+1, \bar{b}, \tilde{x}),\left(M, \bar{b}+\overline{1}_{1}, \tilde{x}\right), \cdots$, $\left(M, \bar{b}+\overline{1}_{i}, \tilde{x}\right), \cdots$, and $\left(M, \bar{b}+\overline{1}_{N}, \tilde{x}\right) \in \Omega$, where $\overline{1}_{i}$ is the vector $(0, \cdots, 0,1,0, \cdots, 0)$ of the same dimension as $\bar{b}$ with the $i$ th element equal to 1 and all other elements equal to 0 . 
Consider a case where $u_{i}(M, \bar{b}, \tilde{x})>\widehat{u}_{i}$ and $u_{j}(M, \bar{b}, \tilde{x})<\widehat{u}_{j}$. Since $u_{i}(M, \bar{b}, \tilde{x})$ is decreasing in $g_{i}$, if $g_{i} \geq g_{j}$, it is useless to swap the locations of two workstations. Otherwise, the swap should be tried to expect the situation $u_{j}>\widehat{u}_{j}$.

\section{Solution Procedure}

A solution procedure to seek the optimal solution $\left(M^{*}, \bar{b}^{*}, \tilde{x}^{*}\right)$ is developed by using the monotonicity properties in the preceding section as follows:

Solution procedure:

Step1: (Initialization)

1.1. Set the search list $\Gamma$ as an empty set.

1.2. Obtain the initial location matrix $\tilde{x}_{L B}$ by using equation (12).

1.3. Set $d_{i}=\sum_{j=1}^{L} \eta_{j} x_{i j}$ and $g_{i}=\sum_{j=1}^{L} \nu_{j} x_{i j}, \quad i=1, \cdots, N$.

1.4. Compute $M_{L B}$ and $\bar{b}_{L B}$ from equations (4) and (6), respectively.

1.5. Put the first node $\left(M_{L B}, \bar{b}_{L B}, \tilde{x}_{L B}\right)$ into $\Gamma$.

1.6. Set the iteration number $I T E=0$.

Step2: (Selection, simulation and evaluation)

2.1. Select the node at which $\Psi(M, \bar{b}, \tilde{x})$ takes the minimum value in $\Gamma$. If more than one candidate attains the minimum value, select the node which enters first in $\Gamma$.

2.2. Compute $\bar{u}=\left(u_{1}, u_{2}, \cdots, u_{N}\right)$ using simulation at the selected node.

2.3. Set $I T E=I T E+1$.

2.4. If $u_{i}(M, \bar{b}, \tilde{x}) \geq \widehat{u}_{i}$ for all $i(i=1, \cdots, N)$, then go to step4. Otherwise go to step3.

Step3: (Branch)

The node selected in step2.1 is referred to as the parent node.

3.1. Make two branch nodes, $(M, \bar{b}+\triangle, \tilde{x})$ and $(M+1, \bar{b}, \tilde{x})$, where $(M, \bar{b}+\triangle, \tilde{x})$ represents the node with $b_{i}=b_{i}+1$ for all $i^{\prime} s$ such that $u_{i}(M, \bar{b}, \tilde{x})<\widehat{u}_{i}$. Put the branch nodes into $\Gamma$.

3.2. In the parent node, machine $i$ is reordered in increasing order of $g_{i}$ and the rearranged machine is denoted by $(i)$.

3.2.1. Set $(i)=1$.

3.2.2. If $u_{(i)} \geq \widehat{u}_{(i)}$, then go to step3.2.5.

3.2.3. Find the maximum $(j)$ that satisfies both $(j)<(i)$ and $u_{(j)}>\widehat{u}_{(j)}$, and make a branch node $\left(M, \bar{b}, \tilde{x}^{\prime}\right)$, where $\tilde{x}^{\prime}$ denotes $\tilde{x}$ with locations $(i)$ and $(j)$ swapped. If such $(j)$ does not exist, go to step3.2.5.

3.2.4. If the cost of the branch node is smaller than the cost of the parent node, put the branch node into $\Gamma$.

3.2.5. Set $(i)=(i)+1$. If $(i)>N$, then go to step3.3. Otherwise go to step3.2.2.

3.3. Put the parent node out of $\Gamma$. Go to step2.

Step4: Print out $\Psi, M, \bar{b}, \tilde{x}, \bar{u}$ and $I T E$, and terminate.

It is clear that if $\widehat{u}_{i}<1-\epsilon$ for a positive value $\epsilon, i=1, \cdots, N$, then the solution procedure converges.

\section{Examples and Computational Results}

In this section, numerical results are given in order to observe the lower bounds and the monotone structure and investigate the efficiency of the proposed procedure. Let $N=$ 
$L=5$. We adopt the AGV loop configuration shown in Figure 1. The distribution of the processing times at machine $i$ is assumed to be the Erlang distribution of phase $k_{i}$ with mean $1 / \mu_{i}$, whose variance $\sigma_{i}^{2}$ and coefficient of variation $Y_{i}$ are given by $1 /\left(k_{i} \mu_{i}^{2}\right)$ and $1 / \sqrt{k_{i}}$, respectively. A manufacturing simulator ROPS II (Nakano et al. 1994) is employed to evaluate the utilization $u_{i}(M, \bar{b}, \tilde{x})(i=1, \cdots, 5)$. The travel times between the dispatching station and possible locations of the workstations are given in Table 1 . The loading and unloading times are assumed to be 20 , respectively.

Table 1: Travel times for possible locations

\begin{tabular}{cccc}
\hline location $j$ & $\nu$ & $\xi$ & $\eta$ \\
\hline 1 & 60 & 60 & 120 \\
\hline 2 & 100 & 60 & 160 \\
\hline 3 & 140 & 20 & 160 \\
\hline 4 & 140 & 100 & 240 \\
\hline 5 & 140 & 60 & 200 \\
\hline
\end{tabular}

At first, suppose that the processing times are deterministic, $\widehat{u}_{i}=0.95(i=1, \cdots, 5)$ and the layout is given by $x_{i i}=1$ and $x_{i j}=0$ for $i \neq j, i=1, \cdots, 5$. Table 2 shows Cases 1 through 8 with different processing times at machines. The pairs of $M_{L B}$ and $\bar{b}_{L B}$ by equations (4) and (6) for the given layout are also shown in Table 2. Figure 2 shows the average machine utilization for different numbers of AGVs in Cases 1 through 6 . For these cases, the buffer capacity $b_{i}(i=1, \cdots, 5)$ is set as three, which is greater than or equal to $b_{i L B}$ shown in Table 2 . The small circles in Figure 2 represent the corresponding $M_{L B}$. It is observed in Figure 2 that $M_{L B}$ satisfies the constraint $u_{i}(M, \bar{b}, \tilde{x})>\widehat{u}_{i}(i=1, \cdots, 5)$ for these cases. For Case 7 of Table 2, the average and minimum machine utilizations for different numbers of AGVs under different buffer capacities are shown in Figures 3 and 4 , respectively. In these figures it is observed that the pair of $M_{L B}=11$ and $\bar{b}_{L B}=(2,1,4,1,2)$ shown as small circles in Figures 3 and 4 achieves $\widehat{u}_{i}, i=1, \cdots, 5$.

Table 2: Example cases and lower bounds for the given layout

\begin{tabular}{|c|c|c|c|c|c|c|c|}
\hline \multirow[b]{2}{*}{ Case } & \multicolumn{5}{|c|}{$1 / \mu_{i}$} & \multirow[b]{2}{*}{$M_{L B}$} & \multirow{2}{*}{$\bar{b}_{L B}$} \\
\hline & 1 & 2 & 3 & 4 & 5 & & \\
\hline 1 & 50 & 50 & 50 & 50 & 50 & 17 & $(22333)$ \\
\hline 2 & 100 & 100 & 100 & 100 & 100 & 9 & $\left(\begin{array}{lllll}1 & 1 & 2 & 2 & 2\end{array}\right)$ \\
\hline 3 & 150 & 150 & 150 & 150 & 150 & 6 & $\left(\begin{array}{llllll}1 & 1 & 1 & 1 & 1\end{array}\right)$ \\
\hline 4 & 200 & 200 & 200 & 200 & 200 & 5 & $\left(\begin{array}{lllll}1 & 1 & 1 & 1 & 1\end{array}\right)$ \\
\hline 5 & 250 & 250 & 250 & 250 & 250 & 4 & $\left(\begin{array}{lllll}1 & 1 & 1 & 1 & 1\end{array}\right)$ \\
\hline 6 & 300 & 300 & 300 & 300 & 300 & 3 & $\left(\begin{array}{llllll}1 & 1 & 1 & 1 & 1\end{array}\right)$ \\
\hline 7 & 50 & 100 & 40 & 200 & 110 & 11 & $\left(\begin{array}{lllll}2 & 1 & 4 & 1 & 2\end{array}\right)$ \\
\hline 8 & 160 & 150 & 40 & 70 & 80 & 12 & $\left(\begin{array}{lllll}1 & 2 & 4 & 4 & 3\end{array}\right)$ \\
\hline
\end{tabular}

Next, numerical results in case of the non-deterministic processing times and the unfixed locations are illustrated. We use the ratios of $1.1: 1: 1$ and $3.1: 1: 10$ for $C_{A}: C_{B}: C_{D}$, and different phases of the Erlang distributions, $k_{i}=1,2,5,10,20,30, \infty$ (deterministic) $i=1, \cdots, 5$. Let $\bar{x}$ be the vector $\left(\sum_{j=1}^{5} j x_{i j}, i=1, \cdots, 5\right)$ in convenience of presentation. The results obtained for Case 7 using the proposed procedure are shown in Table 3. 


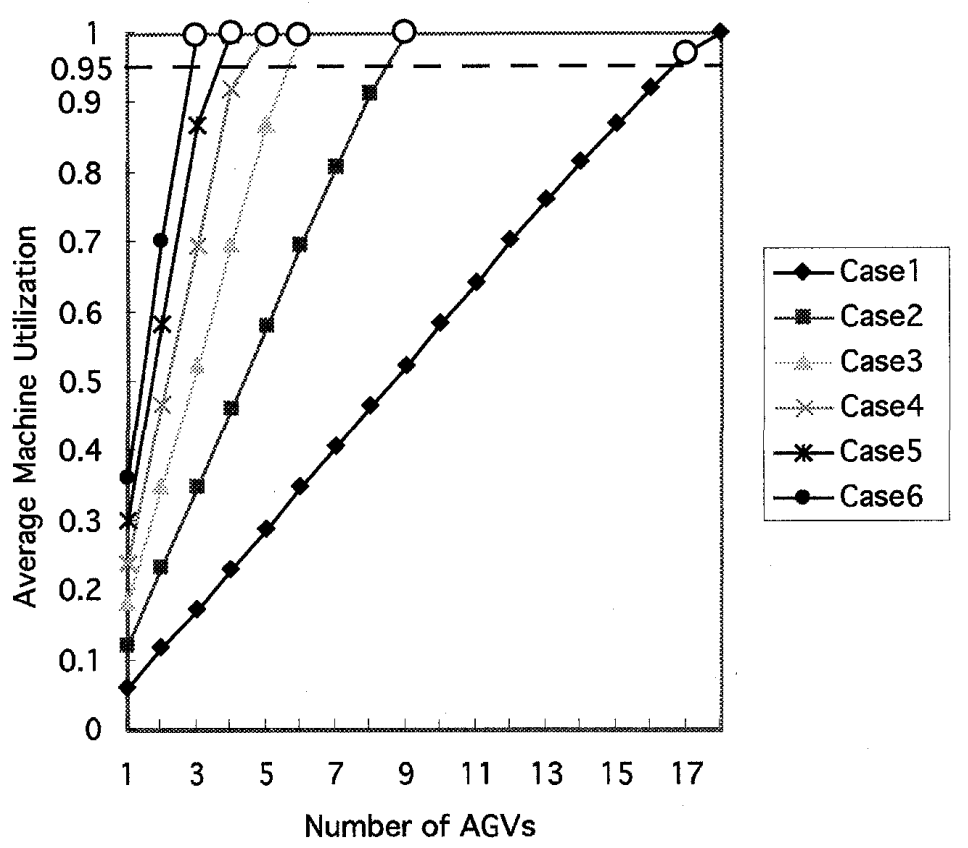

Figure 2: Average machine utilization for different number of AGVs

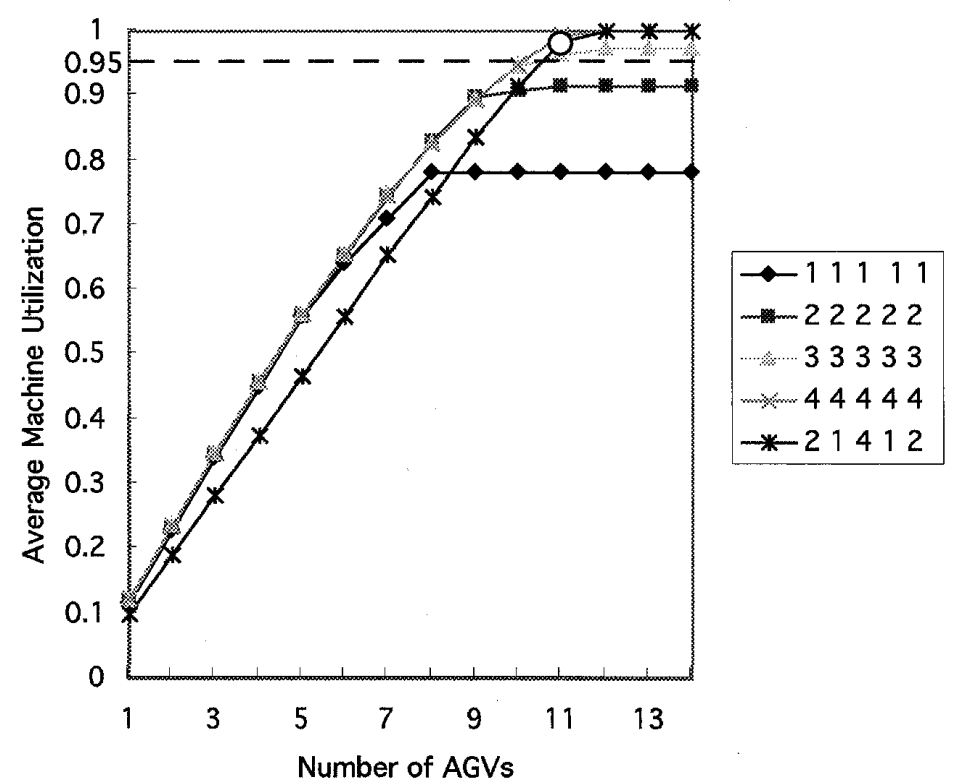

Figure 3: Average machine utilization under different buffer capacities 


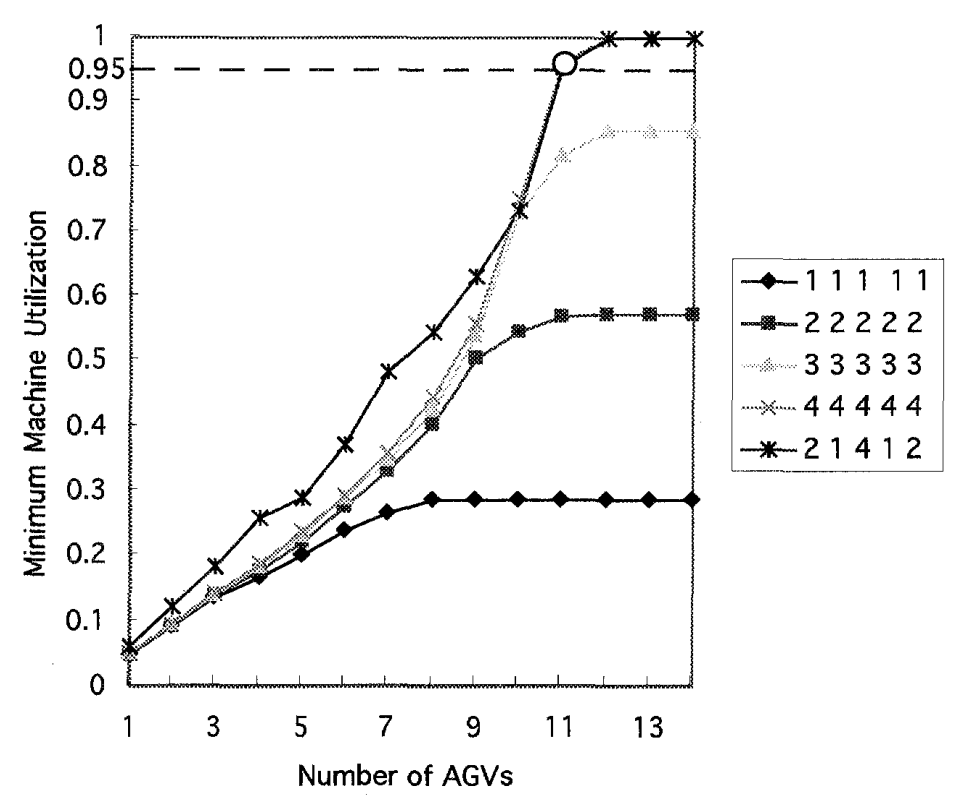

Figure 4: Minimum machine utilization under different buffer capacities

Table 3: Optimal solutions for Case 7

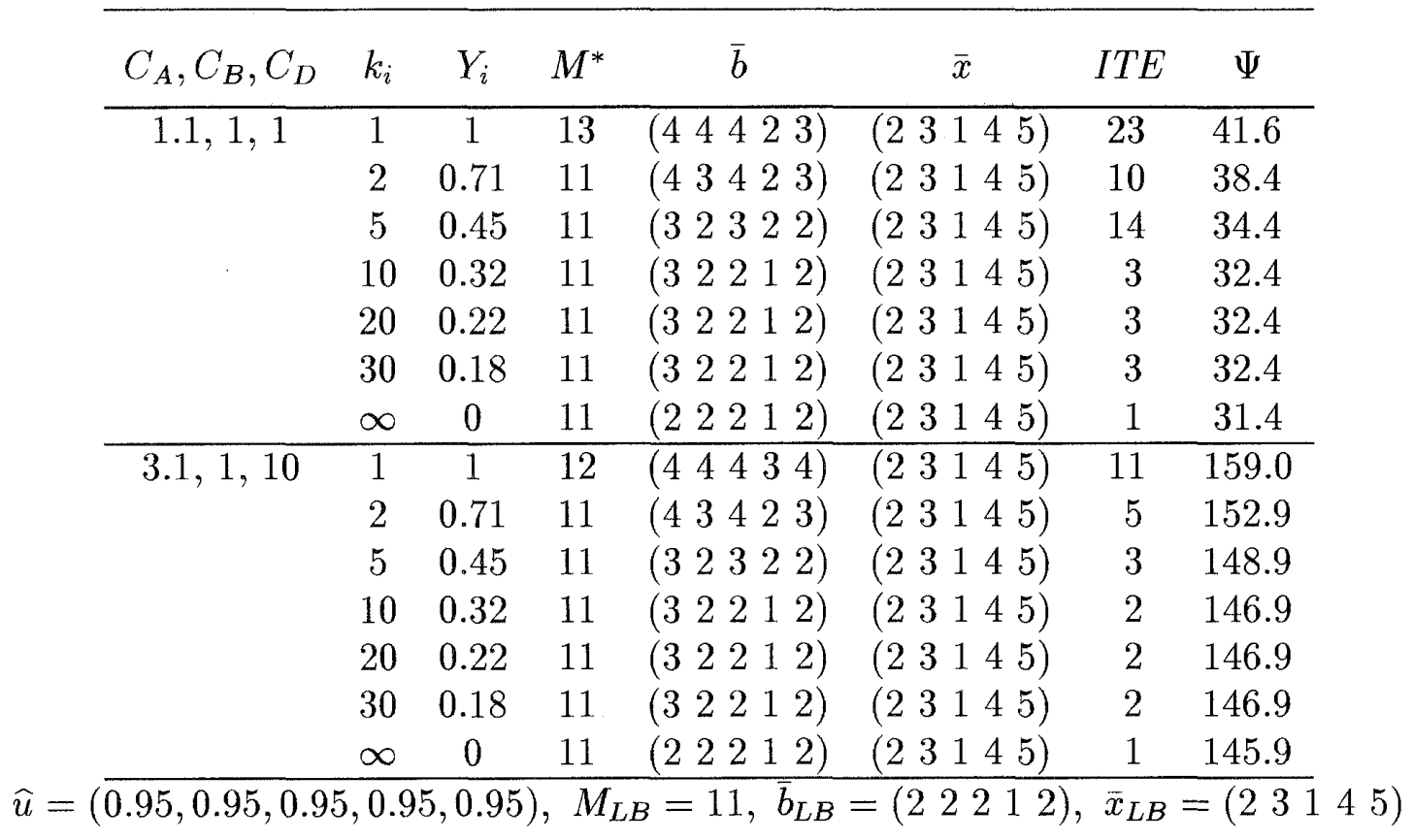


The results suggest that the larger the coefficient of variation $Y_{i}(i=1, \cdots, 5)$ is, the larger the required number of input buffer capacities should be. All iteration numbers ITE are at most three for $Y_{i} \leq 0.32(i=1, \cdots, 5)$ in Tables 3 . This suggests that the proposed procedure is very efficient enough to be used in the design of the AGVS.

Table 4 shows the results for Case 8 with different Erlang phases and different planned machine utilizations. In Table $4, \bar{x}_{L B}$ denotes the $\bar{x}$ with $M=M_{L B}$ and $\bar{b}=\bar{b}_{L B}$, and $\bar{x}^{*}$ denotes the optimal solution of $\bar{x}$. In this table, $\bar{k}$ denotes $\left(k_{1}, \cdots, k_{N}\right)$ and $\widehat{u}$ denotes $\left(\widehat{u}_{1}, \cdots, \widehat{u}_{N}\right)$. It is to be noted that the lower bounds $\left(M_{L B}, \bar{b}_{L B}, \bar{x}_{L B}\right)$ are the optimal solutions in cases of the deterministic processing times in Tables 3 and 4 . This implies that steps 2 and 3 in the proposed procedure can be omitted in case of the deterministic processing times. Then the proposed procedure is non-iterative and the simulation is unnecessary. Hence the proposed procedure is useful in the practical design of the AGVS.

Table 4: Optimal solutions for Case 8

\begin{tabular}{|c|c|c|c|c|c|c|}
\hline $\begin{array}{c}\text { planned machine } \\
\text { utilization }(\widehat{u})\end{array}$ & $\begin{array}{c}\text { Erlang } \\
\text { phases }(\bar{k})\end{array}$ & $M^{*}$ & $\bar{b}^{*}$ & $\bar{x}^{*}$ & ITE & $\Psi$ \\
\hline$\left(\begin{array}{lllll}0.95 & 0.95 & 0.95 & 0.95 & 0.95\end{array}\right)$ & $(\infty \infty \infty \infty \infty)$ & 10 & $\left.\begin{array}{llllll}1 & 1 & 2 & 2 & 2\end{array}\right)$ & $\left(\begin{array}{llllll}4 & 5 & 1 & 2 & 3\end{array}\right)$ & 1 & 135.1 \\
\hline$\left(\begin{array}{lllll}0.9 & 0.8 & 0.7 & 0.6 & 0.5\end{array}\right)$ & $(\infty \infty \infty \infty \infty)$ & 10 & $\left(\begin{array}{lllll}1 & 1 & 2 & 1 & 1\end{array}\right)$ & 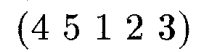 & 1 & 105.9 \\
\hline$\left(\begin{array}{lllll}0.95 & 0.95 & 0.95 & 0.95 & 0.95\end{array}\right)$ & $(5102030 \infty)$ & 10 & $\left(\begin{array}{lllll}2 & 2 & 2 & 2 & 2\end{array}\right)$ & $\left(\begin{array}{lllll}4 & 5 & 1 & 2 & 3\end{array}\right)$ & 2 & 137.1 \\
\hline$\left(\begin{array}{lllll}0.9 & 0.8 & 0.7 & 0.6 & 0.5\end{array}\right)$ & $\left(\begin{array}{lllll}5 & 10 & 20 & 30 & \infty\end{array}\right)$ & 10 & $\left(\begin{array}{lllll}2 & 1 & 2 & 1 & 1\end{array}\right)$ & $\left(\begin{array}{lllll}4 & 5 & 1 & 2 & 3\end{array}\right)$ & 2 & 106.9 \\
\hline
\end{tabular}

\section{Conclusion}

The design problem including multiple decision factors for the AGVS in the JIT environment is considered, and the integrated approach of analytical technique and simulation is proposed to minimize the cost function. The proposed procedure is based on the monotonicity properties of the cost function and the machine utilization in the design factors. It is enough simple to be employed in the practical design of the AGVS. The numerical results show that it becomes non-iterative in case of the deterministic processing times.

In this paper, a workstation includes a machine and an input buffer. In the JIT environment, our results still hold in case of multiple machines in series, by taking the distribution of the processing times at the first machine instead of that of the one machine.

The following conjectures obtained from this study will be true for many other production systems:

(1) In the case where all facilities have deterministic operating times, the lower bound estimated by the simple formula can be optimal as described in this paper.

(2) For production systems with many design factors, the throughput or machine utilization increases with a bottleneck parameter. On the other hand, it behaves insensibly or in a saturated fashion with non-bottleneck parameters. We see it in a sigmoid fashion in Figure 4 and a quadratic concave fashion in Figure 3. For example, in case of

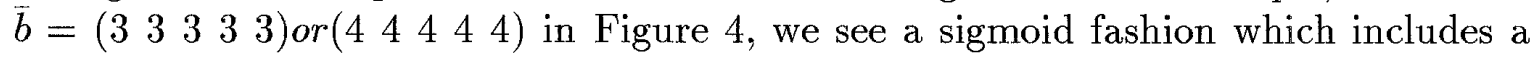
concave increasing situation in $1 \leq M \leq 11$ and a saturated situation in $12 \leq M \leq \infty$. The concave fashion is expected also by Ozden (1988) and Sinriech(1992) from their simulation study.

We believe that many design problems of manufacturing systems may have such monotone structure as described above. The idea of this paper originally came from analyzing 
design processes of experienced factory designers. They often deal with complicated design problems of manufacturing systems today by intuition and hand-calculation. We can learn the above heuristic rules from their experiences.

\section{Acknowledgment}

The authors wish to acknowledge the encouragement given by Dr. Atsushi Danno, Mr. Hiroshi Moribe, and Mr. Tomohiko Yamazaki, TOYOTA CENTRAL R\&D LABS., INC.

\section{References}

[1] J. A. Buzacott, and J. G. Shanthikumar: Stochastic Models of Manufacturing Systems (Prentice-Hall, Englewood Cliffs, New Jersey, 1993).

[2] P. J. Egbelu: The use of non-simulation approaches in estimating vehicle requirements in an automated guided vehicle based transport system. Material Flow, 4 (1987) 17-32.

[3] P. J. Egbelu and J. M. A. Tanchoco: Characterization of automatic guided vehicle dispatching rules. International Journal of Production Research, 31-12 (1984) 359-374.

[4] P. Glasserman and D. D. Yao: Monotone Structure in Discrete-Event Systems (John Wiley \& Sons, Wiley-Interscience, New York, 1994) 105-115.

[5] H. Hwang, S. Y. Kim, and S. M. Moon: Determination of optimum unit load size of the AGV in an electronics assembly production system. International Journal of Production Research, 34-5 (1996) 1293-1306.

[6] M. E. Johnson and M. L. Brandeau: An analytic model for design of a multivehicle automated guided vehicle system. Management Science, 39-12 (1993) 1477-1489.

[7] J. Kim and C. M. Klein: Location of departmental pickup and delivery points for an AGV system. International Journal of Production Research, 34-2 (1996) 407-420.

[8] K. H. Kim and J. M. A. Tanchoco: Economical design of material flow paths. International Journal of Production Research, 31-6 (1993) 1387-1407.

[9] B. Mahadevan and T. T. Narendran: "A hybrid modelling approach to thedesign of an AGV-based material. International Journal of Production Research, 32-9 (1994) 2015-2030.

[10] B. Mahadevan and T. T. Narendran: Estimation of number of AGVs for an FMS: an analytical model. International Journal of Production Research, 31-7 (1993) 1655-1670.

[11] C. J. Malmborg: Heuristic, storage space minimization methods for facility layouts served by looped AGV systems. International Journal of Production Research, 32-11 (1994) 2695-710.

[12] A. W. Marshall and I. Olkin: Inequalities: Theory of Majorization and Its Applications (Academic Press, Harcourt Brace Jovanovich, New York, 1979).

[13] W. L. Maxwell and J. A. Muckstadt: Design of automated guided vehicle systems. IIE Transactions, 14-2 (1982) 114-124.

[14] Y. Monden: Toyota Production System, 2nd ed. (Industrial Engineering and Management Press, Norcross, 1993).

[15] M. Nakano, N. Sugiura, M. Tanaka, and T. Kuno: ROPSII: agent-oriented manufacturing simulator on the basis of robot simulator. Proceedings of Japan-U.S.A. Symposium on Flexible Automation (1994) 201-208.

[16] L. G. Occeña and T. Yokota: Modeling of an automated guided vehicle system (AGVs) in a just-in-time (JIT) environment. International Journal of Production Research, 29-3 (1991) 495-511. 
[17] K. Ohno, K. Nakashima, and M. Kojima: Optimal numbers of two kinds of kanbans in a JIT production system. International Journal of Production Research, 33-5 (1995) $1387-1401$.

[18] M. Ozden: A simulation study of multiple-load-carrying automated guided vehicles in a flexible manufacturing system. International Journal of Production Research, 26-8 (1988) 1353-1366.

[19] D. Sinriech and J. M. A. Tanchoco: An economic model for determining AGV fleet size. International Journal of Production Research, 30-6 (1992) 1255-1268.

[20] J. G. Shantikumar and R. G. Sargent: A unifying view of hybrid simulation/analytic models and modeling. Operations Research, 31 (1983) 1030-1052.

\author{
Masaru Nakano \\ Software Science Laboratory \\ Toyota Central R\&D Labs., Inc. \\ Nagakute, Aichi-ken 480-1192, JAPAN \\ E-mail: nakano@robotics.tytlabs.co.jp
}

